\title{
Distance-Based Routing Strategy for Traffic Transport in Spatial Networks
}

\author{
Wei Huang, Xiang Pan, Xi Yang, and Jianhua Zhang \\ College of Computer Science, Zhejiang University of Technology, Hangzhou 310023, China \\ Correspondence should be addressed to Wei Huang; huangwei@zjut.edu.cn
}

Received 1 August 2013; Accepted 20 September 2013

Academic Editor: Ming Li

Copyright (C) 2013 Wei Huang et al. This is an open access article distributed under the Creative Commons Attribution License, which permits unrestricted use, distribution, and reproduction in any medium, provided the original work is properly cited.

\begin{abstract}
It is well known that routing strategies based on global topological information is not a good choice for the enhancement of traffic throughput in large-scale networks due to the heavy communication cost. On the contrary, acquiring spatial information, such as spatial distances among nodes, is more feasible. In this paper, we propose a novel distance-based routing strategy in spatial scale-free networks, called LDistance strategy. The probability of establishing links among nodes obeys the power-law in the spatial network under study. Compared with the LDegree strategy (Wang et al., 2006) and the mixed strategy (a strategy combining both greedy routing strategy and random routing strategy), results show that our proposed LDistance strategy can further enhance traffic capacity. Besides, the LDistance strategy can also achieve a much shorter delivering time than the LDegree strategy. Analyses reveal that the superiority of our strategy is mainly due to the interdependent relationship between topological and spatial characteristics in spatial scale-free networks. Furthermore, along transporting path in the LDistance strategy, the spatial distance to destination decays more rapidly, and the degrees of routers are higher than those in the LDegree strategy.
\end{abstract}

\section{Introduction}

In the last few years, the analysis and modelling of dynamics in networked systems have attracted much attention in the field of theoretic physics [1-3]. Such networked systems include the Internet, high-way networks, airline networks, and social, biology, and some other infrastructure networks. In some real networks such as the Internet [4], electric-power grid [5], and airline networks [6], each node has its individual precise position in the space and the spatial distances among nodes cannot be arbitrarily neglected. Moreover, the spatial distances among nodes are not identical. In such networks, the network is embedded into a space with some (e.g., Euclidean) metric. This is why people usually call these networks spatial networks [7].

Until now, most previous work only focused on the effects of topological characteristics on dynamical occurring in networks, while the effects of spatial characteristics begin to attract much attention only in recent years. It has been reported that in real networks, the topological and spatial characteristics are closely related [8]. Two nodes close to each other are likely to be connected even though both nodes have low degrees, whereas there may not exist any link between two high-degree nodes far away from each other. For example, in an airline network, nodes represent cities, and two nodes are connected by a link if there is at least one airline between cities corresponding to the two nodes. Two big cities, such as Beijing and New York, even though they are far away from each other, they are still connected with each other. However, it is unlikely that two small cities are connected, unless they are close enough. Empirical studies [8] revealed that the effects of spatial characteristics can play an important role in affecting dynamics on networks [9]. Exploring the effect of spatial characteristics on dynamics may reveal some new and interesting features which cannot be observed when only studying the effect of topological characteristics.

Among different kinds of dynamics on networked systems, transport is a typical kind of dynamics that can be universally observed in real systems. Typical examples include the delivery of information packets in the Internet and airplane flights in airline networks. Much effort has been dedicated to designing efficient routing strategies. Efficient routing strategy can alleviate traffic congestion and enhance transport efficiency $[10,11]$. By now, most studies 
for designing efficient routing strategy are mainly based on topological information or dynamical information in the network (e.g., the number of packets on nodes). Such typical routing strategies include the shortest path routing strategy [12], the local routing strategy based on nodes' degrees [13], the routing strategy integrating both static topological information and dynamical traffic information [14-17], the routing strategy integrating both local and global topological information $[18,19]$, the routing strategy aiming to minimize the maximal node betweenness $[20,21]$, and the routing strategy aiming to find the so-called efficient path [22].

Although routing strategies based on topological information have been extensively studied, routing strategies based on spatial information have so far rarely been considered. Actually, in real communication networks, utilizing global topological information, for example, the shortest path between two nodes, needs to consume much communication cost or even cannot be accessed. For example, in the Internet at autonomous system (AS) level, the shortest path between two nodes may be illegal due to complicated relationship of commercial benefit among internet service providers (ISP). On the contrary, spatial distances among nodes are comparably convenient to obtain. The position of each packet's destination node can be affiliated on the packet itself. Since inquiring neighbors' position in the geographical space does not consume much communication cost for each node, when a packet arrives at a node, the spatial distance between each neighbor and the packet can be easily calculated without consuming much communication cost. It is worth noting that the spatial information can be used for searching a destination node from a source node without global topological information in large-size networks [23]. In [23], the authors performed the greedy searching strategy in scale-free networks. In the greedy searching strategy, each node uses the information of spatial distance to select, as the next hop, closest to the destination in the network. The authors claimed that the most navigable topologies are with small degree exponent of the degree distribution and with strong clustering. Moreover in [24], the authors pointed out that the greedy searching in scale-free networks with strong clustering and power-law node degree distribution $\gamma<3$ find its path with the average scaling as $\ln \ln N$, which is the same as the shortest path length. These reports validate that the information of spatial distance can effectively improve searching efficiency in spatial scale-free networks. In addition, there have also been some reports on the study of spatial ingredients in the fields of communication and computer networks [25-27].

Therefore, in this paper, we propose a novel routing strategy based on the information of spatial distance, to enhance transport efficiency in spatial scale-free networks. Global topological information is not required, and only the local topological information is utilized in our strategy. Therefore, we call our strategy the LDistance (local and based on the information of spatial distance) strategy. To highlight the advantage of spatial information in enhancing transport efficiency, we compare our proposed routing strategy with the local routing strategy based on nodes' degrees [13] (we call it the LDegree strategy in our paper) and the mixed strategy, which is a combination of greedy routing strategy and random routing strategy. Extensive simulations are performed in spatial networks with scale-free structure. Results show that the proposed LDistance routing strategy shows strong superiority over the LDegree strategy and the mixed strategy. Besides, results also show that our proposed LDistance strategy can also achieve a shorter delivering time than the LDegree strategy.

This paper is organized as follows. In Section 2, we present some preliminary work, including the model of spatial networks with scale-free network structure, some backgrounds of our study on traffic problems, and the LDegree strategy proposed in [13]. Then, we present our proposed LDistance routing strategy in spatial networks with scale-free structure. In Section 3, simulation results are presented, followed by detailed analyses and discussions. Finally, conclusions are drawn in Section 4.

\section{Model Description}

In our study, the modelling of traffic transport is based on the spatial network model proposed in [8]. This model reproduces several important features that can be observed in real communication networks, such as the Internet at the autonomous system level and the USA airline network [23]. These features include small-world, scale-free, and strong clustering. It should be mentioned that strong clustering means that spatial information is highly related to the underlying topological information. In this model, the nodes are placed on a circle. Each node is assigned with a random variable representing the node's polar angle, which is evenly distributed in $[0,2 \pi)$. To keep the density of nodes on the circle fixed to 1 , the total number of nodes is proportional to the circle radius $R$. Each node is then assigned with an expected degree $k$, which is drawn from a power-law degree distribution $P(k) \sim k^{-\gamma}$. Then we connect each of the two nodes with the probability $r\left(d ; k, k^{\prime}\right)$ that depends on the geodesic distance $d$ between the two nodes and the nodes' assigned degrees $k$ and $k^{\prime}$. The probability $r\left(d ; k, k^{\prime}\right)$ takes the form:

$$
\begin{gathered}
r\left(d ; k, k^{\prime}\right)=\left(1+\frac{d}{d_{c}}\right)^{-\lambda}=\left(1+\frac{d}{\mu k k^{\prime}}\right)^{-\lambda}, \\
\mu=\frac{(\lambda-1)}{2\langle k\rangle},
\end{gathered}
$$

where $\langle k\rangle$ denotes the average expected degree. Note that the probability follows the power-law and is heavy-tailed $[28,29]$, which is rather critical in computer networks [30]. With this form of connection probability, long-range links are discouraged and short-range links are favored, as we have mentioned in Section 1 . Moreover, the parameter $\lambda$, called clustering strength, controls the significance of spatial distance for establishing new links among nodes. The increasing of $\lambda$ can strengthen the tendency of establishing a new link between two nodes that are close to each other. Therefore, in a network with large $\lambda$, connections appear more frequently among the nodes that are close to each other in the spatial 
space. It can be inferred consequently that strong clustering can easily form in the network with large $\lambda$. The stronger the influence of spatial information on underlying network topology, the more strongly nodes are clustered.

Next, we will describe the model of traffic transport on spatial networks. For simplicity, we treat all nodes in the network as both hosts and routers for generating and delivering packets. At each time step, $M$ packets are generated in the network with randomly chosen sources and destinations (we denote $M$ as the packet generation rate). Note that for each packet, the source node and the destination node must be different. A packet, if its destination is rightly one of the neighbors of the current node where the packet is located, the packet will be directly forwarded to the destination node. Otherwise, the packet is forwarded from one node to another following a given routing strategy. Each node $i$ is assigned with a given delivering capacity $C_{i}$, that is, the maximal number of packets each node can deliver at one time step. Without loss of generosity, we set the delivering capacity for each node equal to 5 in our study. An arrived packet will be placed at the end of the queue if this node already has some packets to be delivered to their respective destinations. Packets in queue work on a FIFO (first in first out) basis. Finally, a packet will be removed from the network once it reaches its destination.

In this study, we focus on the traffic capacity [31] and the average delivering time, to evaluate the efficiency of traffic transport in spatial networks with scale-free structure. The traffic capacity can be defined as the critical value $M_{c}$ of the packet generation rate $M$ and is usually described by an order parameter [31]:

$$
\eta(M)=\lim _{t \rightarrow+\infty} \frac{\langle\Delta W\rangle}{M \Delta t}
$$

where $\langle\Delta W\rangle=W(t+\Delta t)-W(t)$, and $\langle\cdots\rangle$ is the average over time windows of width $\Delta t$. $W(t)$ denotes the total number of packets in the network at time step $t$. The network undergoes a phase transition from a free-flow state to congested state at $M=M_{c}$. Under the condition $M<M_{c}, W(t)=0$ and $\eta=0$, this indicates that the number of generated and removed packets is kept balanced and the network is under free-flow state. On the other hand, when $M>$ $M_{c}$, for packets, the generation rate exceeds the removing rate statistically. Therefore, packets are becoming congested with the elapsing of time steps. Under this condition, $\eta$ is above zero. The designed routing strategy in communication networks should try to maximize the traffic capacity and minimize the average delivering time of packets.

In the LDegree strategy, each node performs a local search among all its direct neighbors. If the packet's destination is found to be not among the neighbors of current node, the packet is delivered to node $i$, one of the neighbors of current node, with probability:

$$
\prod_{i}=\frac{k_{i}^{\alpha}}{\sum_{j} k_{j}^{\alpha}},
$$

where the sum runs over all neighbors of the current node, $k_{i}$ is the degree of node $i$, and $\alpha$ is a tunable parameter. The authors of [13] found that the optimal parameter $\alpha=-1$ can achieve the maximal traffic capacity when the delivering capacity is identical for each node.

In this paper, we shall first compare our proposed LDistance strategy with the LDegree strategy. Based on the spatial network model in [8], when any node receives a packet, the node should easily know the distance between any of its neighbors and the packet's destination node, as we have stated in Section 1. Any global topological information is not needed in our strategy. Let us assume a packet's destination is node $t$. If the packet's destination $t$ is found to be not among the neighbors of current node, the packet is forwarded to the nodes $i$, one of the neighbors of current node, with probability:

$$
\prod_{i}=\frac{d_{i, t}^{\beta}}{\sum_{j} d_{j, t}^{\beta}},
$$

where the sum runs over all neighbors of the current node, and $d_{i, t}$ denotes the geodesic distance between node $i$ and $t$, and $\beta$ is a tunable parameter. If $\beta<0$, it means that packets are inclined to be forwarded to the neighbor with relatively short distance to destination. If $\beta>0$, packets are more likely to be forwarded to the neighbor with relatively long distance to destination. Obviously when $\beta \rightarrow-\infty$, the proposed routing strategy is recovered to the greedy routing strategy; that is, each packet is always forwarded to the neighbor with the smallest geodesic distance to destination.

To further validate the high efficiency of the LDistance strategy, we will also compare the proposed LDistance strategy with another kind of distance-based routing strategy, called the mixed strategy in this paper. The mixed strategy is a mixture of the greedy routing strategy and the random routing strategy. In this mixed strategy, at each time step, we choose the neighbor closest to destination as the next hop with probability $1-\sigma$ and randomly choose a neighbor with probability $\sigma$. It is obvious that when $\sigma=0$, the mixed strategy is recovered to the LDistance strategy with $\beta=-\infty$, that is, the greedy routing strategy. When $\sigma=1$, the mixed strategy becomes a purely random routing strategy.

\section{Result, Analysis, and Relevant Discussions}

In our study, all experiments were performed in the spatial scale-free network model introduced in Section 2 with the average degree $\langle k\rangle=13$ and the network size $N=$ 500. Different values of clustering exponent $\lambda$ and degree exponent $\gamma$ are assigned for the network model under study. We mainly focus on the traffic capacity $M_{c}$ and the average delivering time $T_{\text {ave }}$. High traffic capacity and low delivering time are favored. Note that the delivering time mainly consists of two parts: the first part is the time consumed on transporting path and the second part is the waiting time on routers. Extensive numerical simulations are performed. Each realization of numerical simulations is terminated when transporting dynamics goes into the steady state; that is, the number of total packets becomes constant or only slightly fluctuate with the elapsing of time steps. According to the 
model and relevant parameter settings in our study, the time step $t_{\text {elapse }}=5000$ is enough to ensure that transporting dynamics have been under the steady state for considerable time steps. Each result is the average over 50 different network configurations and 20 different realizations of transporting dynamics for each network configuration.

Figure 1 demonstrates the traffic capacity $M_{c}$ for both LDegree strategy and LDistance strategy under different parameter settings. As we have stated in Section 2, when $\beta<0$, packets are inclined to be forwarded to the neighbor with short distance to destination, which is usually favored in real networks. However, if the value of $\beta$ is set to be too small, for example, when $\beta \rightarrow-\infty$, the LDistance strategy is recovered to the greedy routing strategy. As [24] pointed out, the average length of greedy routing path scales as $\ln \ln N$, which indicates that the greedy routing path does not deviate much from the shortest path. On other hand, it is well known the shortest path routing strategy can supply even lower transport efficiency than the LDegree strategy. Moreover, in the greedy routing strategy, the packet can easily get stuck at nodes without neighbors closer to the destination than themselves, which finally causes an unsuccessful transport, and transport efficiency is heavily affected. Therefore, the value of $\beta$ should be set to be a proper value in the range $\beta<0$ so that transport efficiency has chances to be enhanced maximally.

For each given clustering exponent and degree exponent, the traffic capacity of the LDistance strategy is higher than that of the LDegree strategy, which indicates that utilizing the information of geodesic distance is beneficial for enhancing traffic capacity in spatial scale-free networks. It is well known that the LDegree strategy has proved to show strong superiority over the shortest path routing strategy [13] in enhancing traffic capacity. The LDistance strategy, on the other hand, provides a novel way of enhancing traffic capacity. The superiority of LDistance strategy over LDegree strategy in enhancing traffic capacity can be explained by virtue of two auxiliary figures, that is, Figures 2 and 3. Figure 2 illustrates the relationship between the topological shortest path length $h$ and the resized distance $d_{n}$ between any two distinct nodes for both the spatial network model proposed in [8] and its null model. The null model, which has the same degree distribution as the spatial network model in [8], is generated following the switching algorithm in [32]. In this switching algorithm, starting from the network model in [8], we carry a series of Monte Carlo switching steps whereby a pair of links $(A-B$ and $C-D)$ are chosen randomly, and then the ends are exchange to give $(A-D$ and $B-C)$. Note that the exchanged is performed only when no multilinks or self-links are generated. For the spatial network model in [8], as shown in Figure 2(a), the topological shortest path length monotonously increases with the spatial distance. In the LDistance strategy, choosing the neighbor with shorter distance to the destination means that the chosen neighbor also has a smaller topological shortest path length from the destination. Vice versa, if the neighbor with relatively long distance to the destination is favored to be chosen, then the packets would have to travel over more routers to find their destinations. However, for the null model, as shown in Figure 2(b), we cannot observe any explicit relationship between the topological shortest path length $h$ and the resized distance $d_{n}$ between any two distinct nodes. Forwarding packets to the neighbor with relatively short distance to destination cannot essentially reduce the topological shortest path length to destination in the null model.

Figure 3 illustrates the relationship between the traffic capacity $M_{c}$ and the parameter $\sigma$ under the mixed routing strategy. We only demonstrate the case of $\gamma=3$, but cases with other degree exponents can still lead to much of the same result as Figure 3. Given the fixed $\gamma$ and $\lambda$, the maximal traffic capacity among different values of tunable parameter ( $\alpha$ for the LDegree strategy, $\beta$ for the LDistance strategy, and $\sigma$ for the mixed strategy) is denoted as $M_{c \max }$. To compare the LDegree strategy, the LDistance strategy, and the mixed strategy, we label $M_{c \max }$ of LDistance strategy and LDegree strategy on the $M_{c}$-axis in each panel of Figure 3. Results show explicitly that in the mixed strategy, $M_{c \max }$ can be achieved at some proper point of $\sigma$. More importantly, it can be observed that for any clustering strength $\lambda$, the mixed strategy cannot supply a higher $M_{c \max }$ than our proposed LDistance strategy.

Now we can give a justification that our proposed strategy has its unique advantage in achieving a higher traffic capacity according to both Figures 2 and 3. Firstly, we have mentioned that in the greedy routing strategy, that is, $\beta \rightarrow-\infty$ in the LDistance strategy, packets can easily get stuck on low-degree nodes. To avoid packets getting stuck on lowdegree nodes, packets cannot always choose routers closest to destination in the geographical space. Therefore, by appropriately increasing $\beta$, we can choose the router with the distance to destination a little longer than the minimal distance to destination to escape from getting stuck on lowdegree nodes. From the relationship between the topological shortest path length $h$ and the resized distance $d_{n}$ illustrated in Figure 2, we can further conjecture that the topological shortest path length from the chosen router to destination also becomes more accordingly. Although the little increment of distance to destination increases some more hops on transporting path, the chance of getting stuck on low-degree nodes can be reduced, and therefore, traffic capacity can be enhanced. However, if the value of $\beta$ is increased excessively, the router with the distance to destination much longer than the minimal distance to destination is likely to be chosen. Consequently, the topological shortest path length from the chosen router to destination can hardly decay along transporting path and therefore traffic capacity can hardly be enhanced. Secondly, the LDegree strategy is distanceagnostic. Along transporting path of LDegree strategy, the distance to destination can hardly decay as fast as that of LDistance strategy, which will be validated in the following part of this paper. Thirdly, in the mixed strategy, to reduce the chance of getting stuck on low-degree nodes, random strategy is involved in the mixed strategy. However, although the incorporating of random strategy can somewhat reduce the chance of getting stuck, the random strategy evidently has more chances to forward packets to the neighbor with rather long distance to destination than our proposed LDistance strategy with optimal $\beta$. We will verify it in the following 


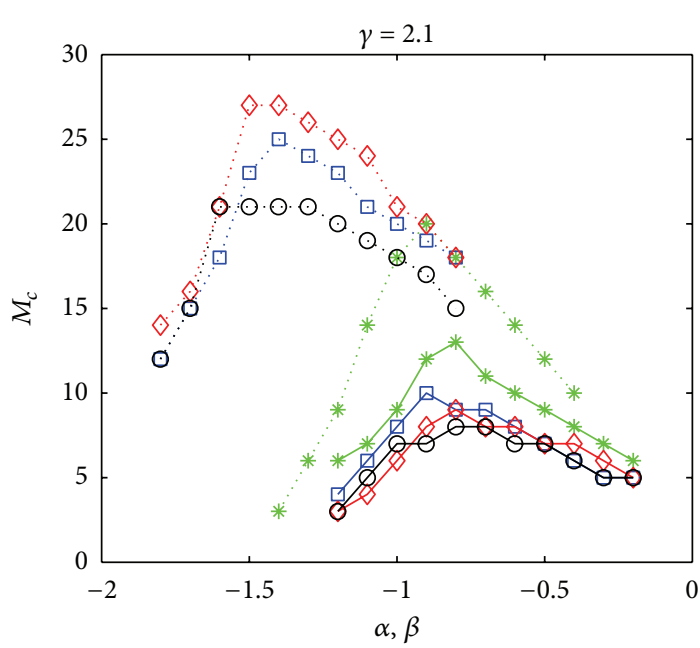

(a)

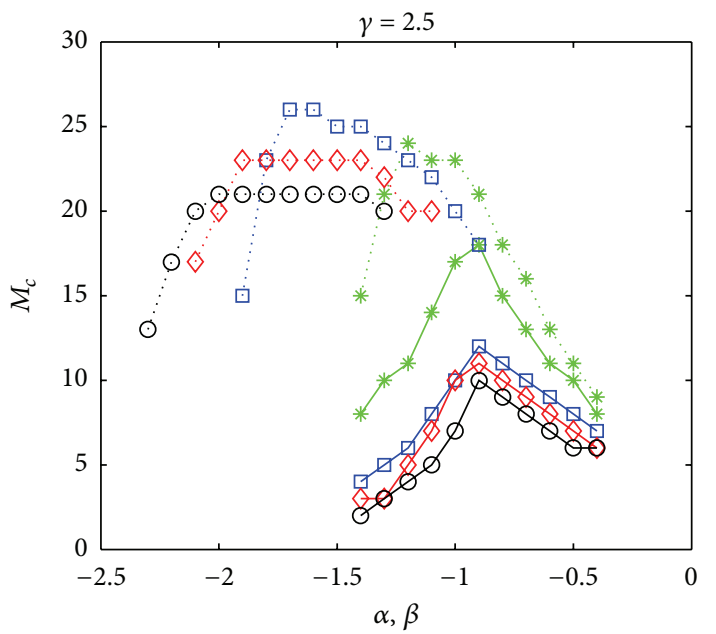

(c)

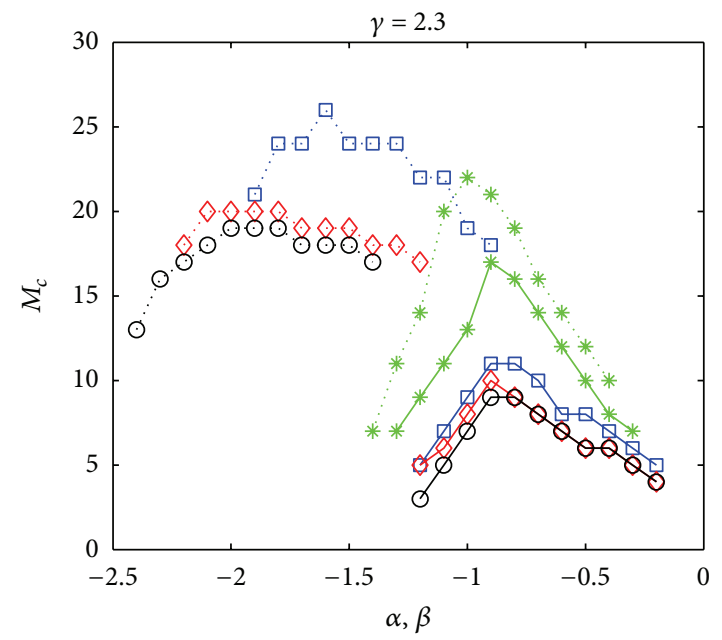

(b)

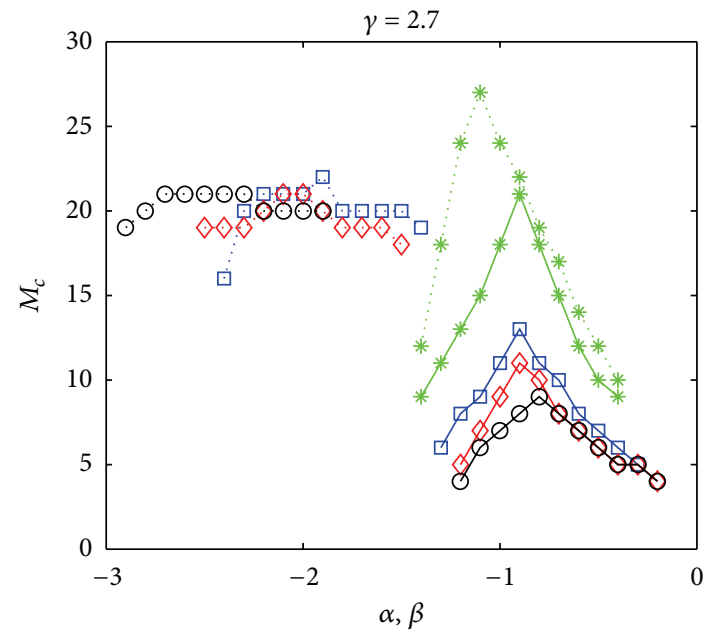

(d)

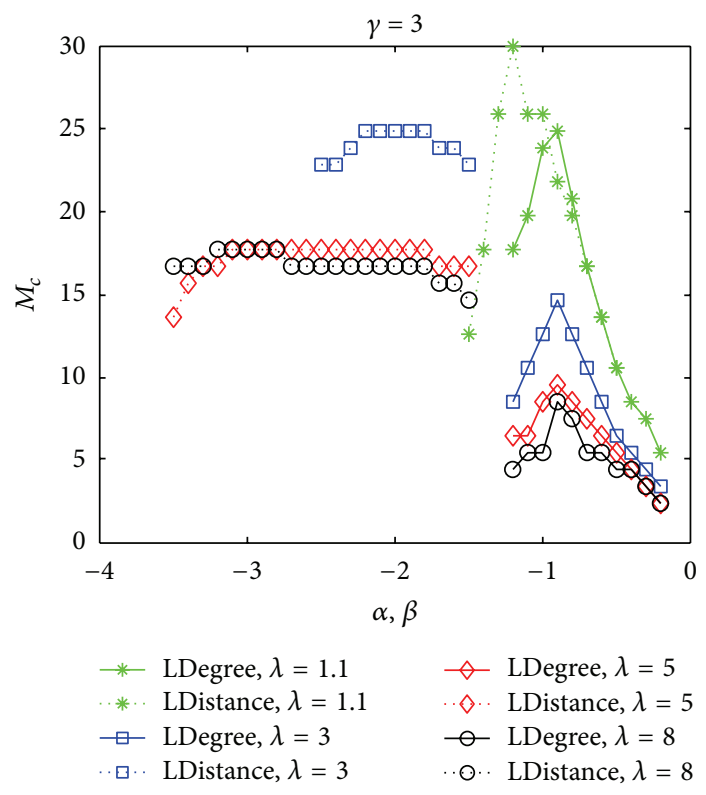

(e)

Figure 1: (Color online) The relationship between traffic capacity $M_{c}$ and tunable parameters ( $\alpha$ for LDegree strategy and $\beta$ for LDistance strategy) under networks with different degree exponent $\gamma$ and clustering strength $\lambda$. 


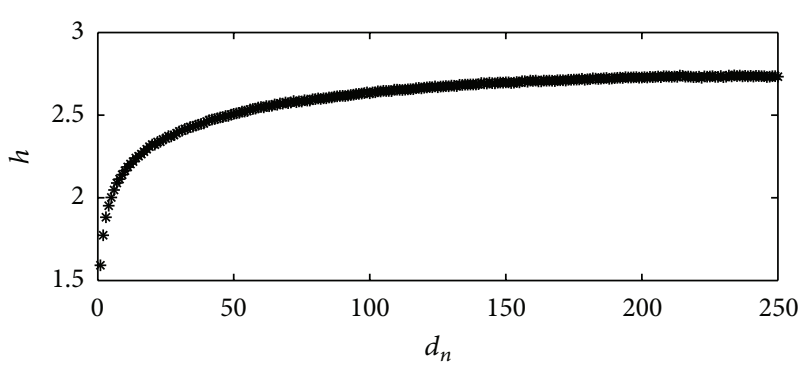

(a) Spatial network model

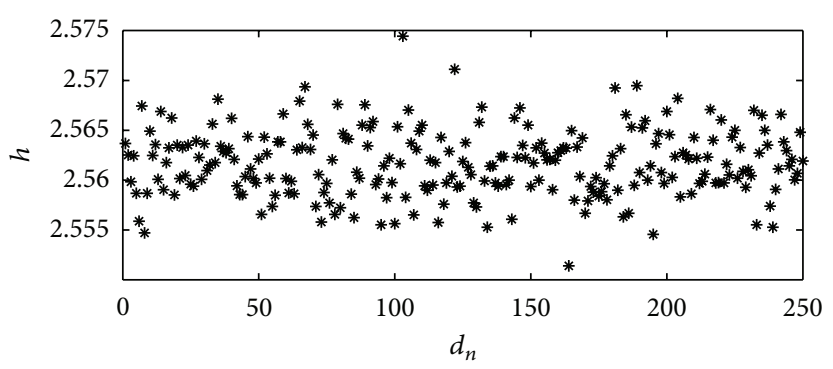

(b) Null model of spatial network model

Figure 2: (Color online) The relationship between the topological shortest path length $h$ versus the resized distance $d_{n}$ between any two nodes for both the spatial network model in [8] and its null model. Here, in the resized distance $d_{n}=d / d_{\text {nei }}, d$ denotes the actual geodesic distance and $d_{\text {nes }}$ denotes the geodesic distance between two neighboring nodes in the geographical space. The degree exponent is set to 3 and the clustering strength in the spatial network model in [8] is set to 1.1 .

part of this paper. Therefore, in the mixed strategy, it is still likely that packets have to travel over more routers to arrive at destinations, which is of course not beneficial for the enhancement of traffic capacity.

By now, we can conjecture that, due to the interdependent relationship between network topology and underlying geographical space, we can only utilize spatial information to achieve high traffic capacities. Note that the acquisition of spatial information is comparably easier and more convenient than that of global topological information, especially in large-size networks. Therefore, our strategy is practically useful in real large-size networks because strategies based on global topological information are inconvenient, or even impossible, to be put into use in real large-size networks.

We have also studied the effect of clustering strength $\lambda$ on traffic capacity for the LDistance strategy. From Figure 1, we can observe that the value of $\beta$ at which $M_{c \max }$ is achieved is decreased with the enhancement of $\lambda$. For example, when $\gamma=3$, the value of $\beta$ at which $M_{c \max }$ is achieved is decreased from -1.1 to around -3 when $\lambda$ rises up to 8 from 1.1. This observed result can be explained as follows. In [23], the authors studied the navigability problem in the spatial scale-free network model proposed in [8]. In their study, they introduced an important navigability parameter, the success ratio $p_{s}$, defined as the percentage of successful paths. Here, unsuccessful paths are those that get stuck on nodes without neighbors closer to destination in the space than themselves, which indicates that packets can hardly arrive at their destinations. The authors pointed out in [23] that, give the fixed degree exponent $\gamma, p_{s}$ increases with networks' clustering strength $\lambda$, which indicates that packets are more likely to get stuck on low-degree nodes in networks with weaker clustering. Therefore, in networks with weaker clustering, to reduce the chance of getting stuck along transporting path, the stringency of packets being forwarded to nodes closest to destinations in the geographical space should be loosened; that is, $\beta$ should be increased. As a result, the success ratio $p_{s}$ can be increased, and therefore, $M_{c \max }$ can be enhanced.

Next, we will concerned with the average delivering time of packets for all strategies under study. Figure 4 illustrates the average delivering time $T_{\text {ave }}$ with the packet generation rate equal to traffic capacity. We only present the case with the degree exponent $\gamma=3$, but the results from cases with other values of $\gamma$ do not deviate too much from the case of $\gamma=3$. Figure 4(a) illustrates the delivering time for both LDistance and LDegree strategies, and the case of LDistance strategy is zoomed in in Figure 4(b). Moreover, the result of the mixture strategy is also shown in the subgraph of Figure 4(b). It can be observed that the minimal $T_{\text {ave }}$ (for a fixed clustering strength $\lambda$ ) achieved from the LDistance strategy does not deviate too much from the mixed strategy under each given clustering strength. However, as Figure 4 shows, both of the two distance-based strategies deliver a much shorter delivering time than the LDegree strategy, which provides another evidence that the performance of our proposed strategy is superior the the LDegree strategy. Moreover, for both the LDistance and the mixed strategies, we find that the impact of clustering strength $\lambda$ on the average delivering time $T_{\text {ave }}$ is not so obvious. However, in the LDegree strategy, $T_{\text {ave }}$ shows strong dependence on $\lambda$. From Figure 4, we have observed that weak clustering induces shorter delivering time. Recalling that the traffic capacities of networks with weak clustering are generally large in the LDegree strategy, we can thus conclude that in the LDegree strategy, transport of packets is more efficient in networks with weak clustering.

From the above analysis, we have found that, as compared to the LDegree strategy, our proposed LDistance strategy can further enhance traffic capacity and shorten average delivering time. To further explore the differences of LDegree and LDistance strategies, we will next compare the structural and spatial characteristics of routers along transporting path for both routing strategies. Figure 5 shows the average resized distance $d_{n}$ to destination and the average degree $k$ of routers along transporting path under the optimal condition; that is, the traffic capacity achieves its maximal value given the predefined $\gamma$ and $\lambda$ for the LDegree strategy, the LDistance strategy, and the mixed strategy. We only demonstrate the case of networks with $(\gamma=3, \lambda=1.1)$, but similar conclusions can also be drawn from other cases with different $\gamma$ and $\lambda$. Figures 5(a) and 5(b) clearly illustrate that, under the optimal condition with the traffic capacity maximized, the length of transporting path in the LDistance strategy is far less than 


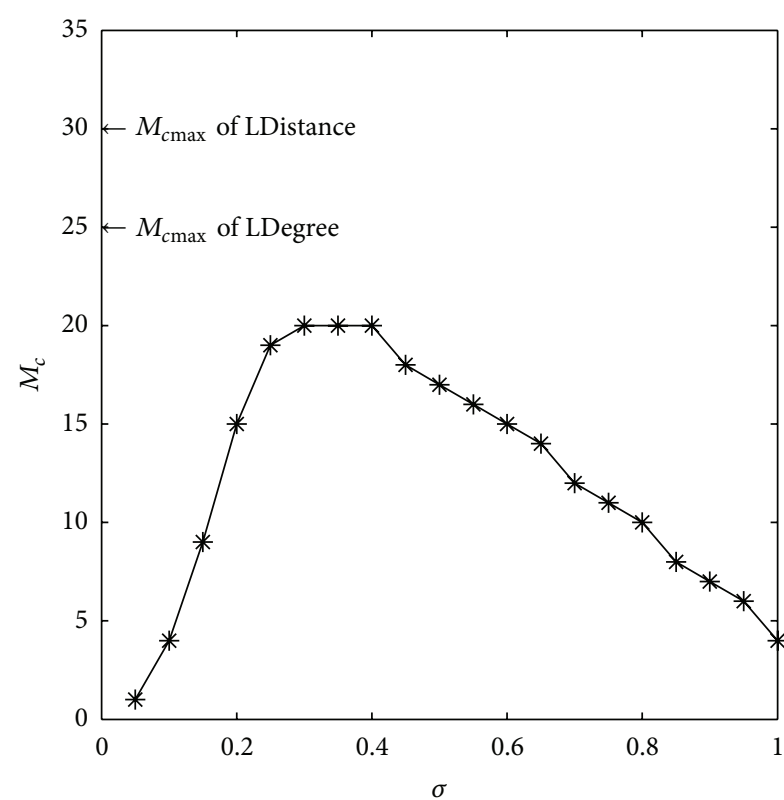

(a) $\lambda=1.1$

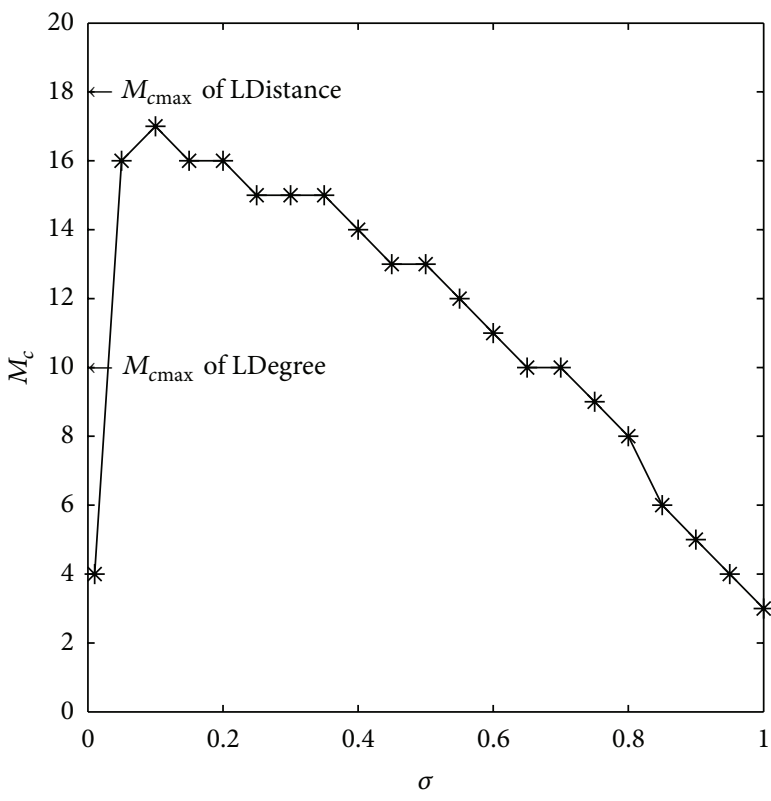

(c) $\lambda=5$

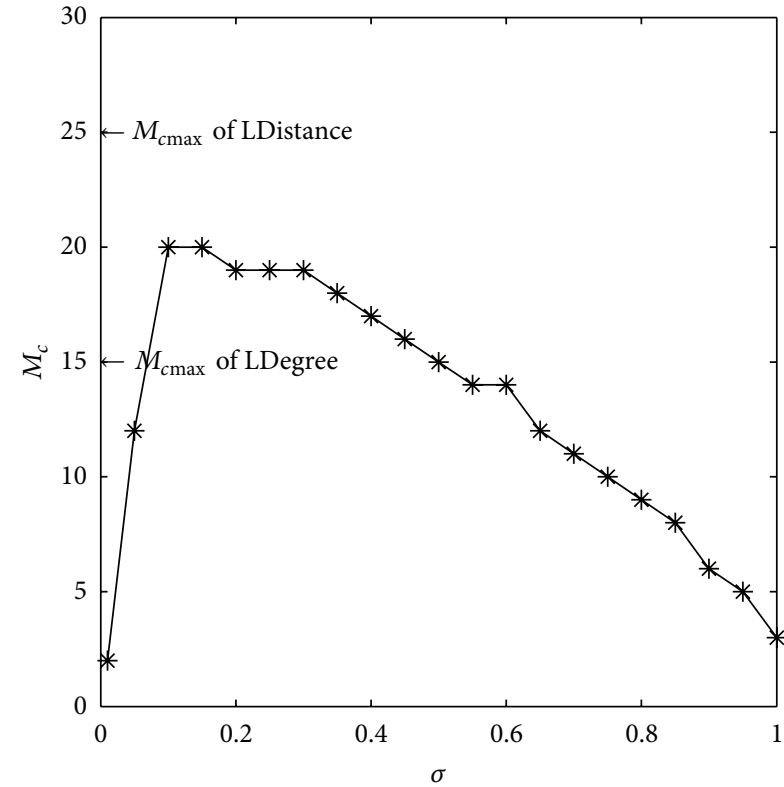

(b) $\lambda=3$

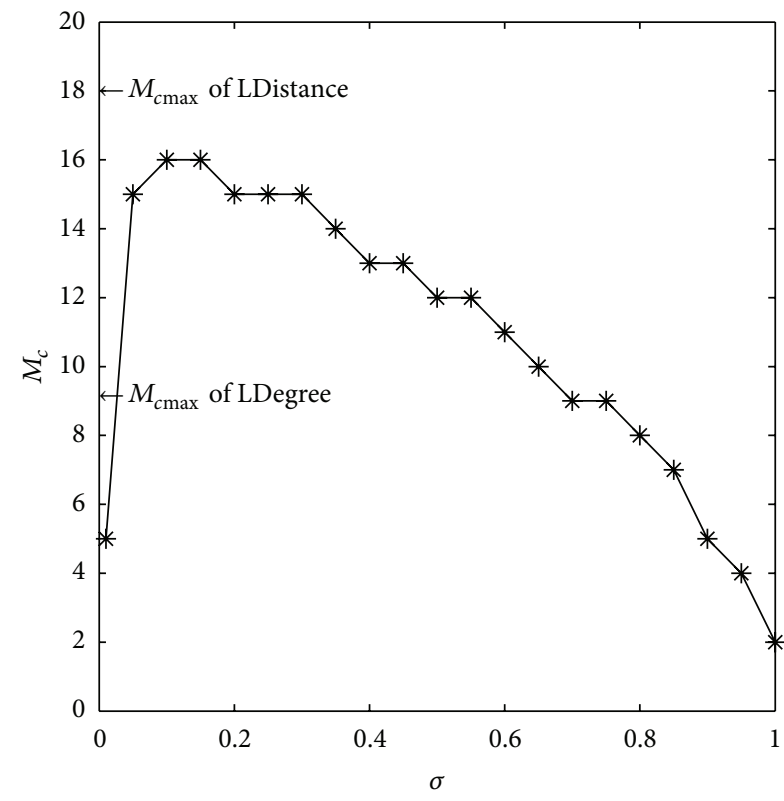

(d) $\lambda=8$

Figure 3: The relationship between traffic capacity $M_{c}$ and tunable parameters $\sigma$ for the mixed strategy under networks with fixed degree exponent $\gamma=3$ and different clustering strength $\lambda$.

that in the LDegree strategy and is slightly less than that in the mixed strategy. We can see under the network with $\gamma=3$ and $\lambda=1.1$ that the average length of transporting path is around 9 under the optimal $\beta_{c}=-1.2$ with the traffic capacity maximized in the LDistance strategy. The average length of transporting path increases to 10 under the optimal condition $\sigma=0.45$ in the mixed strategy. However, in the LDegree strategy, the average length of transporting path rises up to 60 under the optimal condition $\alpha_{c}=-0.9$ with the traffic capacity maximized. We have pointed out that compared to our proposed strategy, the mixed strategy has more chance to forward packets to the neighbor with very long distance to destination. Therefore, in the mixed strategy, the transporting path is longer than that in the LDistance strategy, which is clearly illustrated in Figure 5(a).

Moreover, from Figure 5(a), we can observe that in the LDegree strategy, along transporting path towards destination, the distances of most routers to destination are long and keep almost unchanged. Only at the end of transporting path, the distance to destination decays rapidly until packets finally find their destinations. This result reveals that in the LDegree strategy, except those at the end of transporting path, most deliveries seem not to be as effective as we regarded previously. On the contrary, in the LDistance strategy, the 


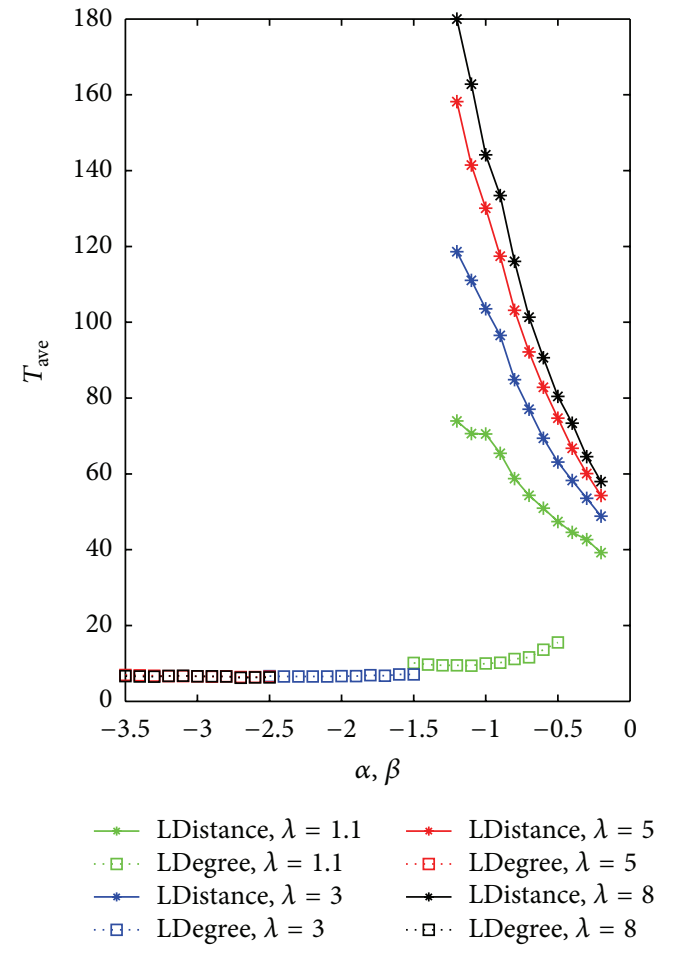

(a)

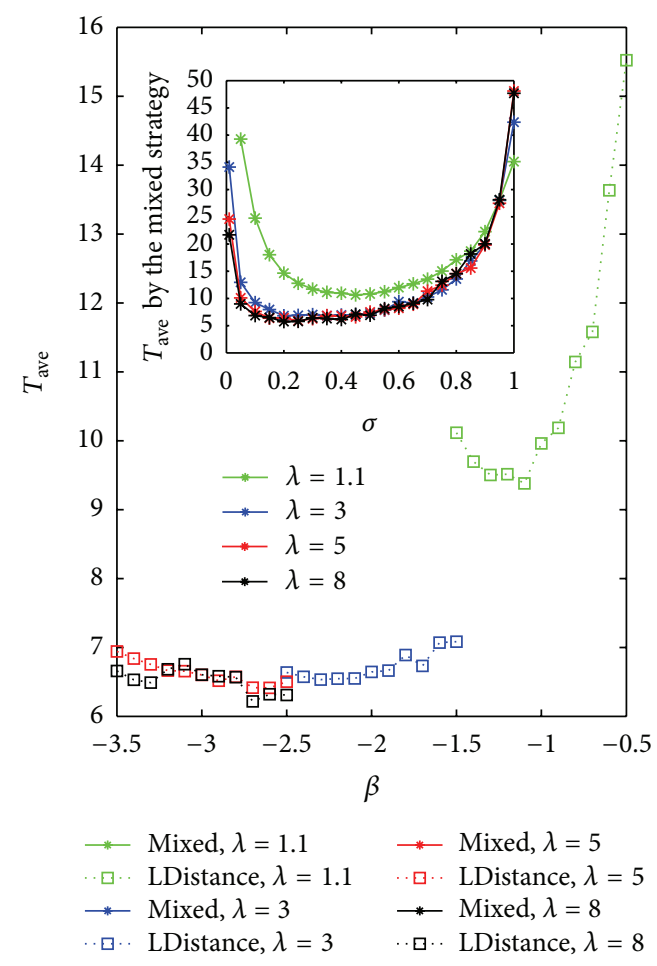

(b)

Figure 4: (Color online) (a) The relationship between average delivering time $T_{\text {ave }}$ and tunable parameters ( $\alpha$ for LDegree strategy and $\beta$ for LDistance strategy) under different clustering strengths on networks with degree exponent $\gamma=3$. For each fixed network, the packet generation rate is set to the same value as the traffic capacity. (b) The case of LDistance strategy is zoomed in. The subgraph of (b) illustrates the average delivering time for the mixed strategy.

distance of routers to destination keeps on decreasing from the beginning of transporting path, which indicates that in the LDistance strategy, each delivery can be considered to be "valuable" for packets being forwarded towards destinations. On the other hand, as can be observed from Figure 5(b), the routers along transporting path in the LDistance strategy evidently have larger degrees than those in the LDegree strategy. In the LDegree strategy, most routers on transporting path have relatively low degrees, except that when the packet arrives at one neighbor of destination. Figure 5(b) reveals that this neighbor usually has very high degrees. In the LDistance strategy, along transporting path, the degrees of routers firstly rise up to rather high values. These high-degree nodes encountered on the transporting path are due to the relationship between a node's characteristics distance scale and the nodes' spatial distance to destination [24]. The closer the chosen nodes' spatial distance to destination, the higher the nodes' degree is. After travelling over these high-degree nodes, the degrees of routers along the following transporting path decrease. At last, the packet arrives at one neighbor (with large degree) of destination once again. The existing LDegree strategy aims to reduce packets' accumulation on high-degree node by dispersing more packets on low-degree nodes. However, our proposed LDistance strategy indicates that the way of dispersing packets on low-degree nodes in the LDegree strategy is far from optimal. The degrees of routers on transporting path need not be so mall as in the LDegree strategy. Increasing the degree of routers on transporting path, as in our proposed LDistance strategy, can help packets find their destinations more quickly.

To further reveal the topological and spatial characteristics of routers on transporting path in the LDistance strategy, we have performed the same simulations as Figure 5, but with different settings of $\beta$. First, Figure 6(a) illustrates the relationship between the average number of hops of transporting path and $\beta$. It shows clearly that the transporting path with the least number of hops is achieved around $\beta \approx$ -0.9 . Above $\beta \approx-0.9$, transporting paths are potentially analogous to those of random search, and therefore, the lengths of transporting paths are lengthened. Figures 6(b) and $6(\mathrm{c})$ repeat the simulations of Figure 5 for the LDistance strategy, but with different values of $\beta$ above $\beta \approx-0.9$. It can be observed that when $\beta$ gets close to $\beta \approx-0.9$, the distance to destination decays more sharply, but the degrees of routers along transporting path do not deviate from each other for different $\beta$. Once again, in Figures 6(d) and 6(e), the simulations of Figure 5 for the LDistance strategy are repeated again with the $\beta$ below -0.9 . When $\beta$ moves away from -0.9 towards $-\infty$, the LDistance strategy is more like the greedy routing strategy, and the interdependence between a nodes' degree and the characteristics scale of spatial distances that the node covers by its connections also becomes stronger. In Figure 6(e), we find that as $\beta$ decreases towards $-\infty$ from $\beta \approx-0.9$, the degree of the 


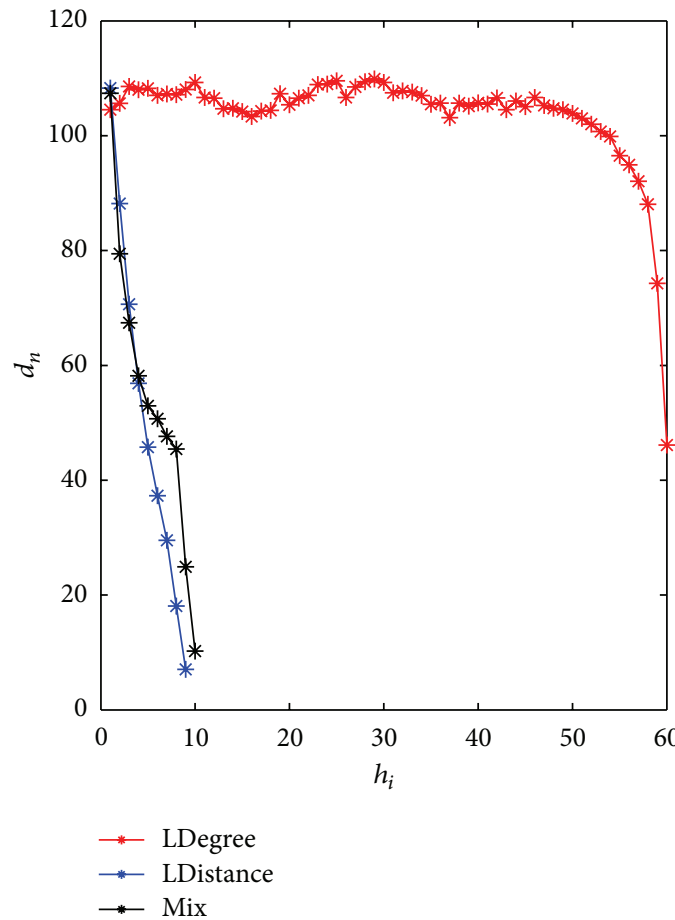

(a)

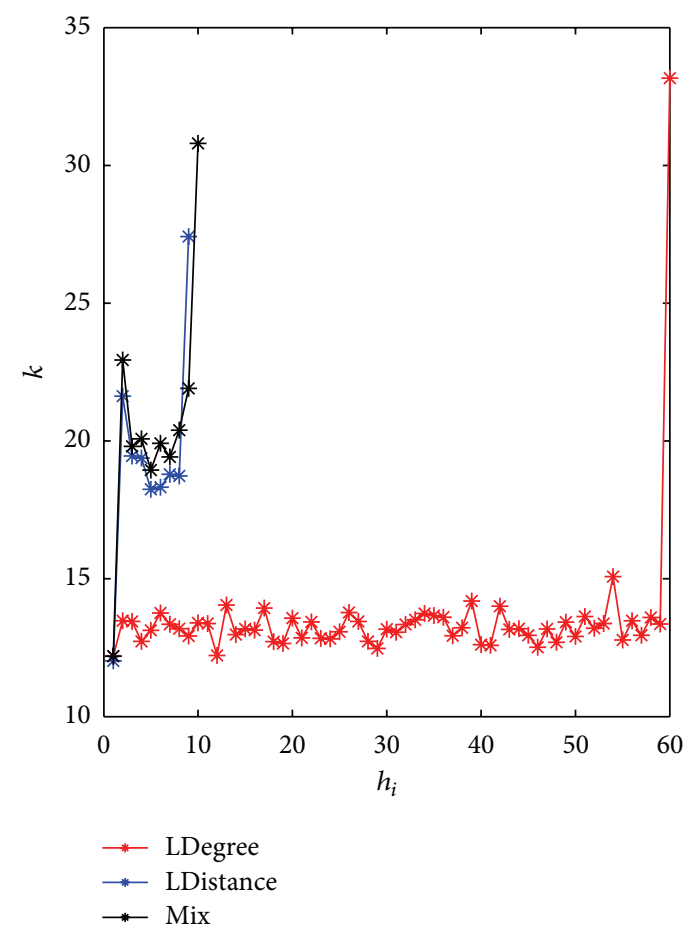

(b)

FIGURE 5: (Color online) Resized distance $d_{n}$ to destination (a) and degree $k$ (b) along transporting path for the LDegree strategy, the LDistance strategy and the mixed strategy. The horizontal axis labels the index $h_{i}$ of routers along transporting path. The tunable parameters are set to $\alpha_{c}=-0.9$ in the LDegree strategy, $\beta_{c}=-1.2$ in the LDistance strategy, and $\sigma=0.45$ because traffic capacities are maximized at these values under the given values of degree exponent $\gamma=3$ and clustering strength $\lambda=1.1$.

first encountered node from the source node becomes higher. Consequently, more packets can accumulate on high-degree nodes and block free flowing of traffic transport. This might be another reason why the traffic capacity becomes decreased as $\beta$ decreases towards minus infinity from $\beta \approx-0.9$, as illustrated in Figure 1(a). Besides, Figure 6(d) also reveals an interesting observation. As $\beta$ decreases towards minus infinity, spatial distance to destination along transporting path decays more sharply. However, under the network with very low $\beta$, for example, $\beta=-2$, when packets are delivered to nodes close to destinations in the geographical space, packets can easily get stuck on low-degree nodes. As a result, packets keep on wandering around low-degree nodes for a rather long time, and the total delivering time is heavily lengthened.

\section{Discussion}

The main motivation of this work stems from the scalability difficulty encountered in real large-size communication networks such as the Internet and the high-way network. Accessing global topological information (e.g., the shortest path between any two nodes) is not an easy task in largesize network due to the rapid growing communication and information processing overhead. Therefore, in current days, it is usually not convenient, or even unfeasible, to design routing strategies based on global topological information. Comparatively, spatial distances among nodes in real networks are relatively easier to be accessed. Then it is feasible and practical to utilize spatial information to design efficient strategies in real large-size communication networks.

In this paper, we propose a novel routing strategy, called the LDistance strategy, which is based on the information of spatial distances among nodes. To evaluate the performances of our proposed LDistance strategy, we compare our proposed LDistance strategy with the local routing strategy based on nodes' degrees proposed in [13] (called the LDegree strategy in this paper) and the mixed strategy, which combines the idea of both greedy routing strategy and random routing strategy. Numerical simulations demonstrate that our proposed LDistance strategy can supply a higher traffic capacity and a shorter delivering time. A reasonable explanation for the superiority of the LDistance strategy over the LDegree strategy is the interdependent relationship between topological and spatial characteristics; that is, the average topological shortest path length between any two nodes increases with the average spatial distance between the two nodes. When a packet is delivered to the neighbor closer to destination in the geographical space, the average shortest path length between the chosen neighbor and destination is also lower. On the other hand, the inclination of packets being forwarded to nodes closest to destination should also not be too strong; otherwise, packets can easily get stuck on low-degree nodes and transport efficiency can be heavily degraded. Due to the incorporating of random strategy in the mixed strategy, packets have more chance to be delivered to the neighbor with longer spatial distance to destination as 


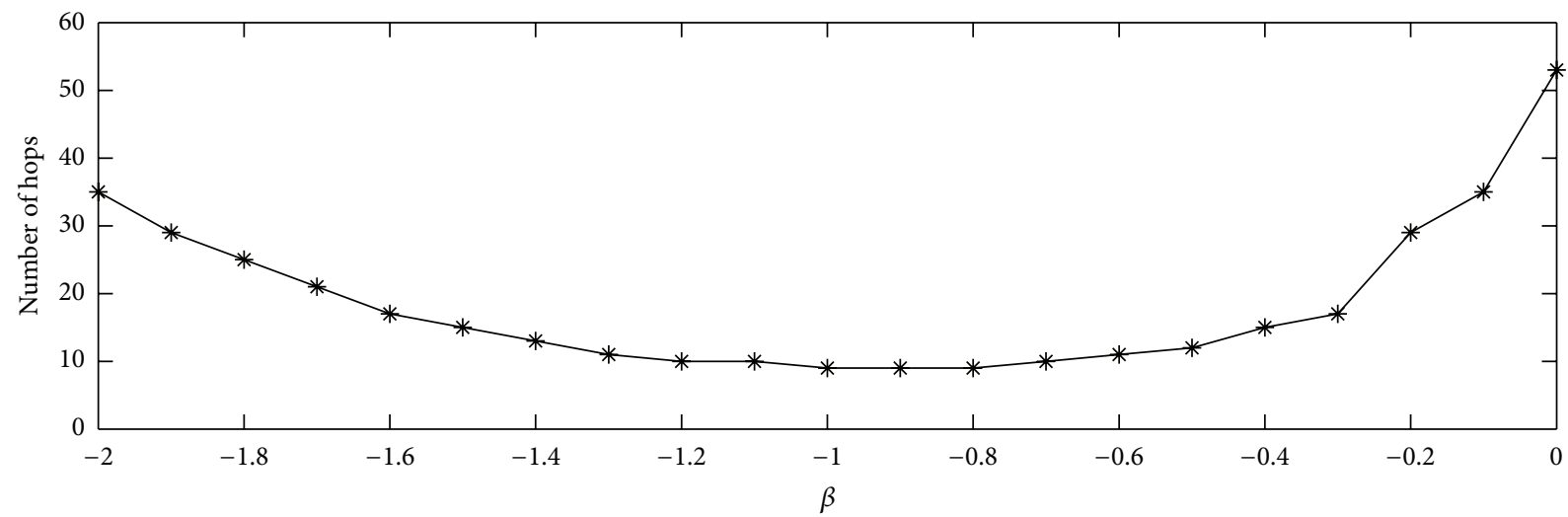

(a)
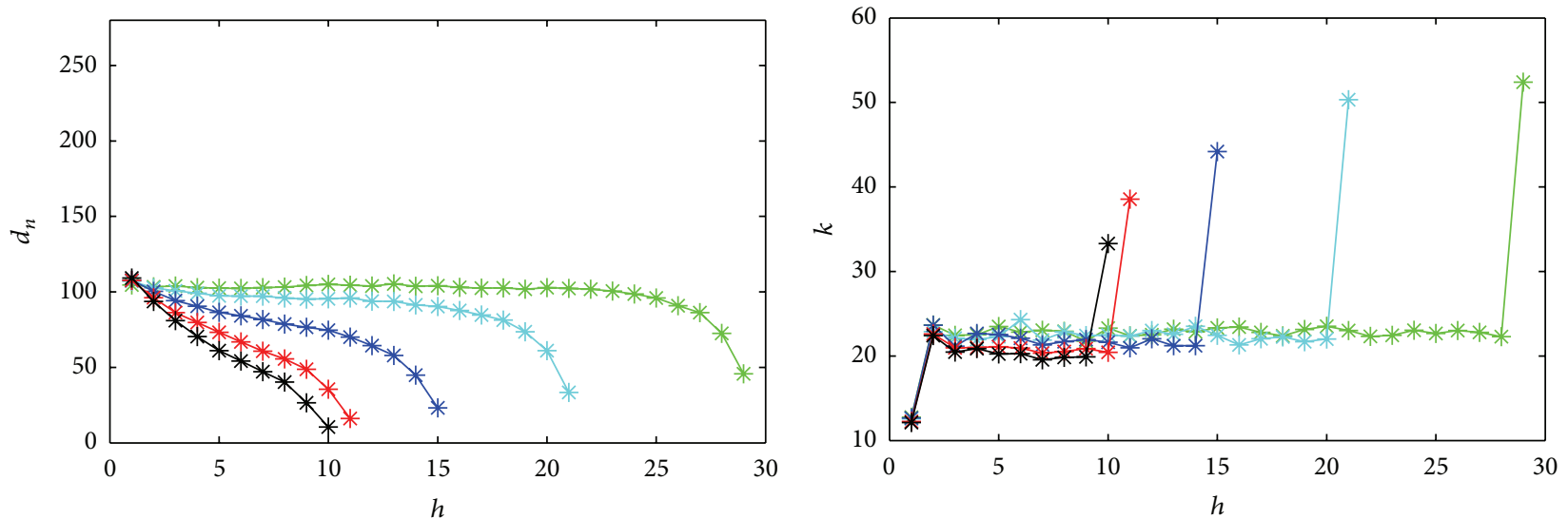

$$
\begin{aligned}
& \rightarrow-\beta=-0.1 \\
& \because-\beta=-0.3 \\
& \rightarrow-\beta=-0.5
\end{aligned}
$$$$
\rightarrow-\beta=-0.7
$$$$
\rightarrow \beta=-0.9
$$

(b)

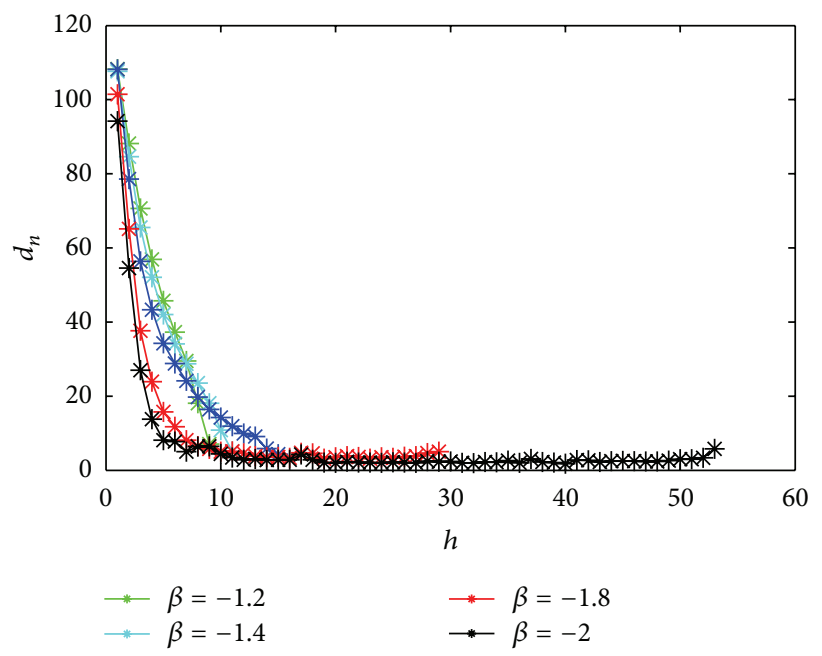

$$
\begin{aligned}
& \because \beta=-0.1 \\
& \because \beta=-0.3 \\
& \because \beta=-0.5
\end{aligned}
$$$$
\rightarrow-\beta=-0.7
$$$$
\rightarrow \beta=-0.9
$$

(c)

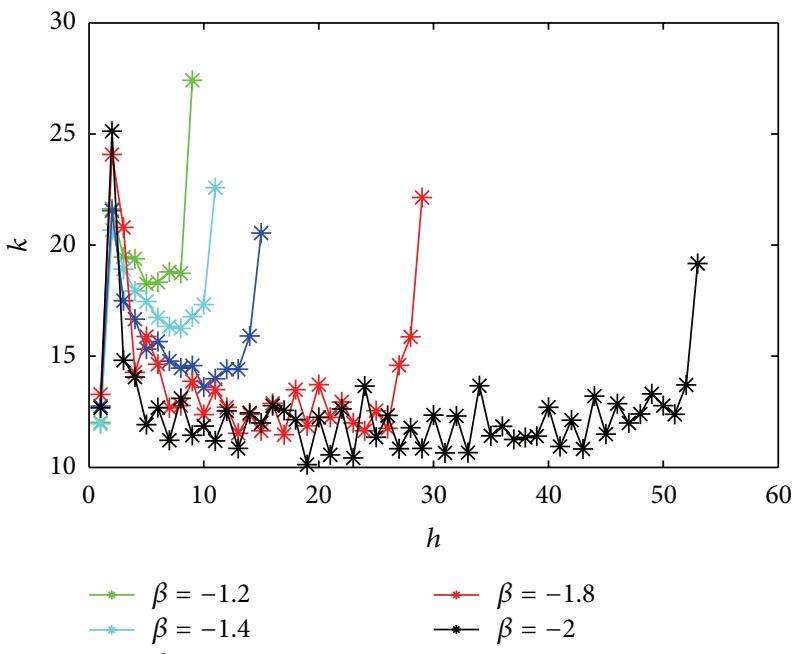

(d)

(e)

Figure 6: (Color online) (a) Number of hops of transporting path versus $\beta$ in the LDistance strategy. (b) and (c) The changing of $d_{n}$ and $k$ versus $h_{i}$ for the LDistance strategy with $\beta$ above $\beta \approx-0.9$. (d) and (e) The changing of $d_{n}$ and $k$ versus $h_{i}$ for the LDistance strategy with $\beta$ below $\beta \approx-0.9$. All simulations were performed in the network with $\gamma=3$ and $\lambda=1$.1. 
compared to the LDistance strategy, which is not beneficial for the enhancement of traffic capacity. Regarding the easy accessing of spatial information in large-size networks, we can conclude that our proposed LDistance strategy can be put into use in real large-size networks to achieve high transport efficiency.

We have also observed that along transporting path in the LDegree strategy, the spatial distance $d$ to destination and the degree $k$ keep almost did not change on most routers before packets arrive at the node very close to their destinations. Therefore, most deliveries are not so valuable for successful transport of packets to destination because spatial distance to destination does not show evident decrease on most routers along transporting path. On the contrary, in the LDistance strategy, along transporting path, the spatial distance to destination monotonously decreases, which means that each time of delivery is valuable for packets approaching destination. Furthermore, we have also noticed that, given the fixed network structure, when the maximal traffic capacities are achieved for both LDegree and LDistance strategies, the degrees of routers along transporting path in the LDistance strategy are higher than those in the LDegree strategy. This result indicates that, to enhance traffic capacity, the way of disseminating packets on low-degree nodes cannot deliver so nice performances as was evaluated previously in the LDegree strategy. Choosing routers with degrees higher than the degrees of chosen routers in the LDegree strategy can actually further improve transport efficiency.

There still remains much room for further studying traffic problems in spatial networks. For example, considering that the theory of network calculus and the technology of leaky bucket have similar goal as our study, we are about to take into account the traffic bound [33] and the bound of packet delay [34] in our further work. We believe that this study is of great importance in real large-size networks, especially in current society with system size increasing explosively. Furthermore, the utilizing of spatial information for designing routing strategies in transport systems can provide more hints for other applications such as information navigation and virus spreading in spatial networks.

\section{Acknowledgments}

This work was supported by the Natural Science Foundation of Zhejiang University of Technology (1101119021408, R1110679), the National Natural Science Foundation of China under Grant nos. 61201074 and 61173096, the Qianjiang Talents Project (QJD1202010), and Doctoral Fund of Ministry of Education of China (20113317110001).

\section{References}

[1] R. Albert and A.-L. Barabási, "Statistical mechanics of complex networks," Reviews of Modern Physics, vol. 74, no. 1, pp. 47-97, 2002.

[2] S. N. Dorogovtsev and J. F. F. Mendes, "Evolution of networks," Advances in Physics, vol. 51, no. 4, pp. 1079-1187, 2002.

[3] M. E. J. Newman, "The structure and function of complex networks," SIAM Review, vol. 45, no. 2, pp. 167-256, 2003.
[4] R. Pastor-Satorras and A. Vespignani, Evolution and Structure of the Internet: A Statistical Physics Approach, Cambridge University Press, Cambridge, UK, 2004.

[5] R. Albert, I. Albert, and G. L. Nakarado, "Structural vulnerability of the North American power grid," Physical Review E, vol. 69, no. 2, Article ID 025103, 4 pages, 2004.

[6] R. Guimerà, S. Mossa, A. Turtschi, and L. A. N. Amaral, "The worldwide air transportation network: Anomalous centrality, community structure, and cities' global roles," Proceedings of the National Academy of Sciences of the United States of America, vol. 102, no. 22, pp. 7794-7799, 2005.

[7] M. Barthélemy, "Spatial networks," Physics Reports, vol. 499, no. 1-3, pp. 1-101, 2011.

[8] M. Ángeles Serrano, D. Krioukov, and M. Boguñá, "Selfsimilarity of complex networks and hidden metric spaces," Physical Review Letters, vol. 100, no. 7, Article ID 078701, 2008.

[9] H. Yang, Y. Nie, A. Zeng, Y. Fan, Y. Hu, and Z. Di, "Scaling properties in spatial networks and their effects on topology and traffic dynamics," Europhysics Letters, vol. 89, no. 5, Article ID 58002, 2010.

[10] B.-H. Wang and T. Zhou, "Traffic flow and efficient routing on scale-free networks: a survey," Journal of the Korean Physical Society, vol. 50, no. 1 I, pp. 134-141, 2007.

[11] S. Chen, W. Huang, C. Cattani, and G. Altieri, "Traffic dynamics on complex networks: a survey," Mathematical Problems in Engineering, vol. 2012, Article ID 732698, 2012.

[12] R. Pastor-Satorras, A. Vázquez, and A. Vespignani, "Dynamical and correlation properties of the internet," Physical Review Letters, vol. 87, no. 25, Article ID 258701, pp. 258701/1-258701/4, 2001.

[13] W.-X. Wang, B.-H. Wang, C.-Y. Yin, Y.-B. Xie, and T. Zhou, "Traffic dynamics based on local routing protocol on a scalefree network," Physical Review E, vol. 73, no. 2, Article ID 026111, 2006.

[14] Z. Y. Chen and X. F. Wang, "Effects of network structure and routing strategy on network capacity," Physical Review E, vol. 73, no. 3, Article ID 036107, 2006.

[15] W. X. Wang, C. Y. Yin, G. Yan, and B. H. Wang, "Integrating local static and dynamic information for routing traffic," Physical Review E, vol. 74, no. 1, Article ID 016101, 2006.

[16] P. Echenique, J. Gómez-Gardeñes, and Y. Moreno, "Improved routing strategies for Internet traffic delivery," Physical Review E, vol. 70, no. 5, Article ID 056105, pp. 1-56105, 2004.

[17] P. Echenique, J. Gómez-gardeñes, and Y. Moreno, "Dynamics of jamming transitions in complex networks," Europhysics Letters, vol. 71, no. 2, pp. 325-331, 2005.

[18] Z. Wu, G. Peng, W. Wong, and K. Yeung, "Improved routing strategies for data traffic in scale-free networks," Journal of Statistical Mechanics, Article ID P11002, 2008.

[19] W. Huang and T. W. S. Chow, "Investigation of both local and global topological ingredients on transport efficiency in scalefree networks," Chaos, vol. 19, no. 4, Article ID 043124, 2009.

[20] B. Danila, Y. Yu, J. A. Marsh, and K. E. Bassler, "Optimal transport on complex networks," Physical Review E, vol. 74, no. 4, Article ID 046106, 2006.

[21] B. Danila, Y. Yu, J. A. Marsh, and K. E. Bassler, "Transport optimization on complex networks," Chaos, vol. 17, no. 2, Article ID 026102, 9 pages, 2007.

[22] G. Yan, T. Zhou, B. Hu, Z. Q. Fu, and B. H. Wang, "Efficient routing on complex networks," Physical Review E, vol. 73, no. 4, Article ID 046108, 2006. 
[23] M. Boguñá, D. Krioukov, and K. C. Claffy, "Navigability of complex networks," Nature Physics, vol. 5, no. 1, pp. 74-80, 2009.

[24] M. Boguñá and D. Krioukov, "Navigating ultrasmall worlds in ultrashort time," Physical Review Letters, vol. 102, no. 5, Article ID 058701, 2009.

[25] K. Tutschku and P. Tran-Gia, "Spatial traffic estimation and characterization for mobile communication network design," IEEE Journal on Selected Areas in Communications, vol. 16, no. 5, pp. 804-811, 1998.

[26] W. Li, M. Canini, A. W. Moore, and R. Bolla, "Efficient application identification and the temporal and spatial stability of classification schema," Computer Networks, vol. 53, no. 6, pp. 790-809, 2009.

[27] S. Uhlig, "On the complexity of Internet traffic dynamics on its topology," Telecommunication Systems, vol. 43, no. 3-4, pp. 167180, 2010.

[28] R. J. Adler, R. E. Feldman, and M. S. Taqqu, Practical Guide to Heavy Tails: Statistical Techniques and Applications, Birkhäuser, Boston, Mass, USA, 1998.

[29] M. Li and W. Zhao, "On $1 / f$ noise," Mathematical Problems in Engineering, vol. 2012, Article ID 673648, 23 pages, 2012.

[30] R. G. Clegg, C. Di Cairano-Gilfedder, and S. Zhou, "A critical look at power law modelling of the Internet," Computer Communications, vol. 33, no. 3, pp. 259-268, 2010.

[31] A. Arenas, A. Díaz-Guilera, and R. Guimerà, "Communication in networks with hierarchical branching," Physical Review Letters, vol. 86, no. 14, pp. 3196-3199, 2001.

[32] M. E. Newman, "Assortative mixing in networks," Physical Review Letters, vol. 89, no. 20, Article ID 208701, 2002.

[33] M. Li and W. Zhao, "Representation of a stochastic traffic bound," IEEE Transactions on Parallel and Distributed Systems, vol. 21, no. 9, pp. 1368-1372, 2010.

[34] M. Li, W. Zhao, and C. Cattani, "Delay bound: fractal traffic passes through network servers," Mathematical Problems in Engineering, vol. 2013, Article ID 157636, 2013. 


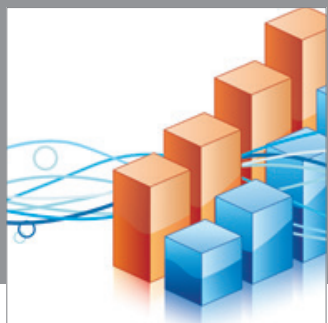

Advances in

Operations Research

mansans

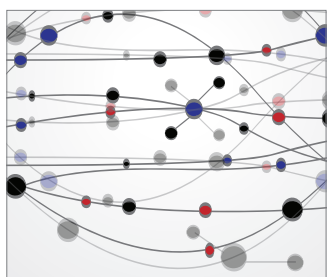

The Scientific World Journal
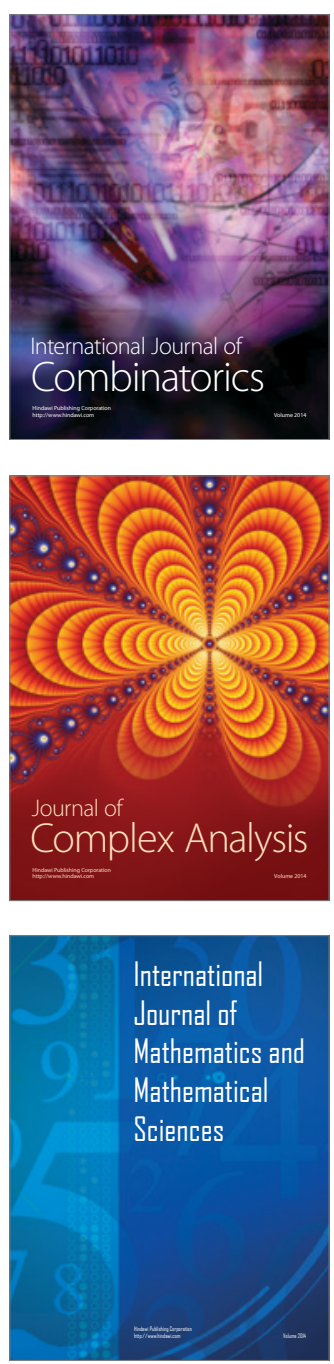
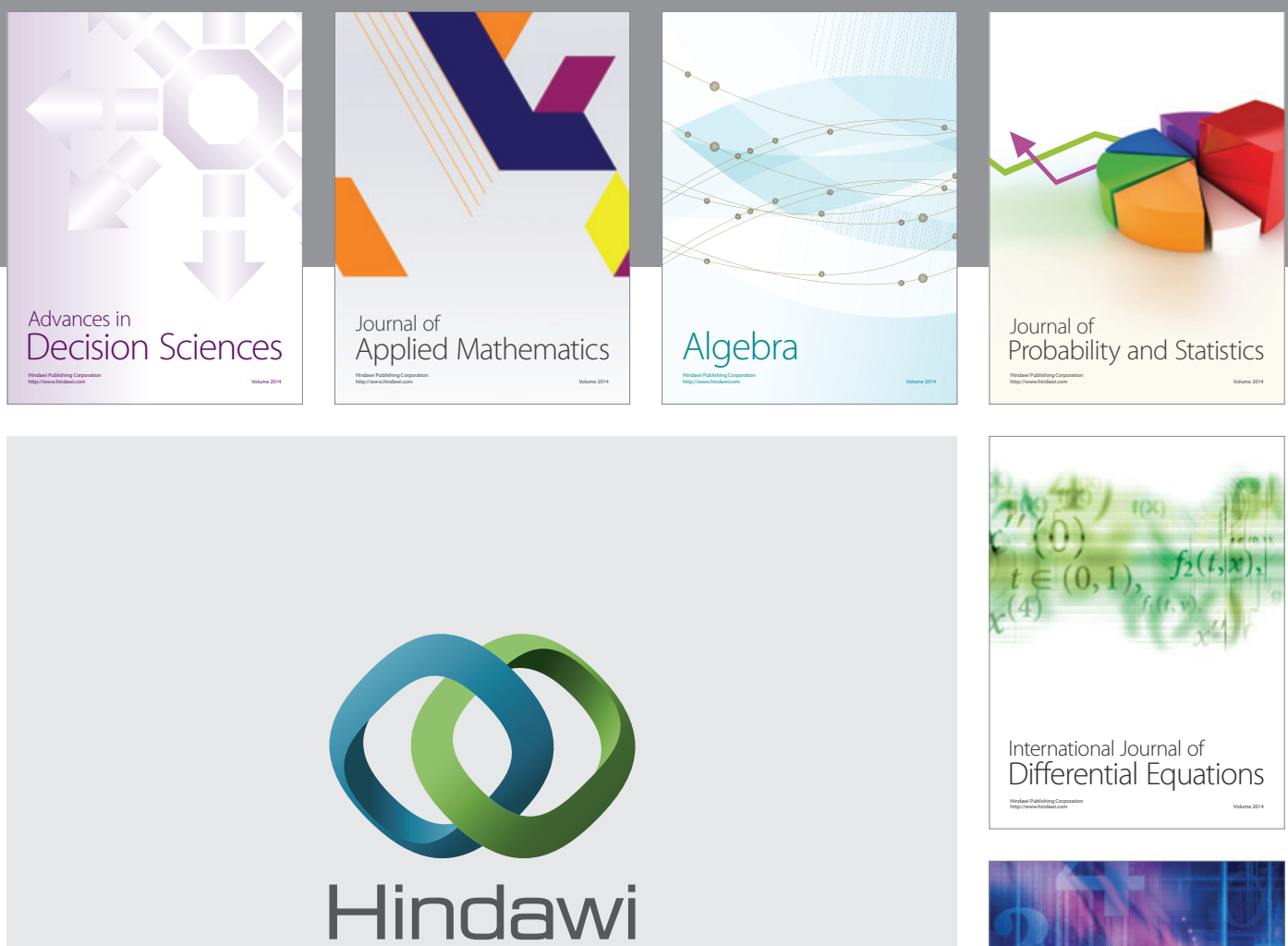

Submit your manuscripts at http://www.hindawi.com
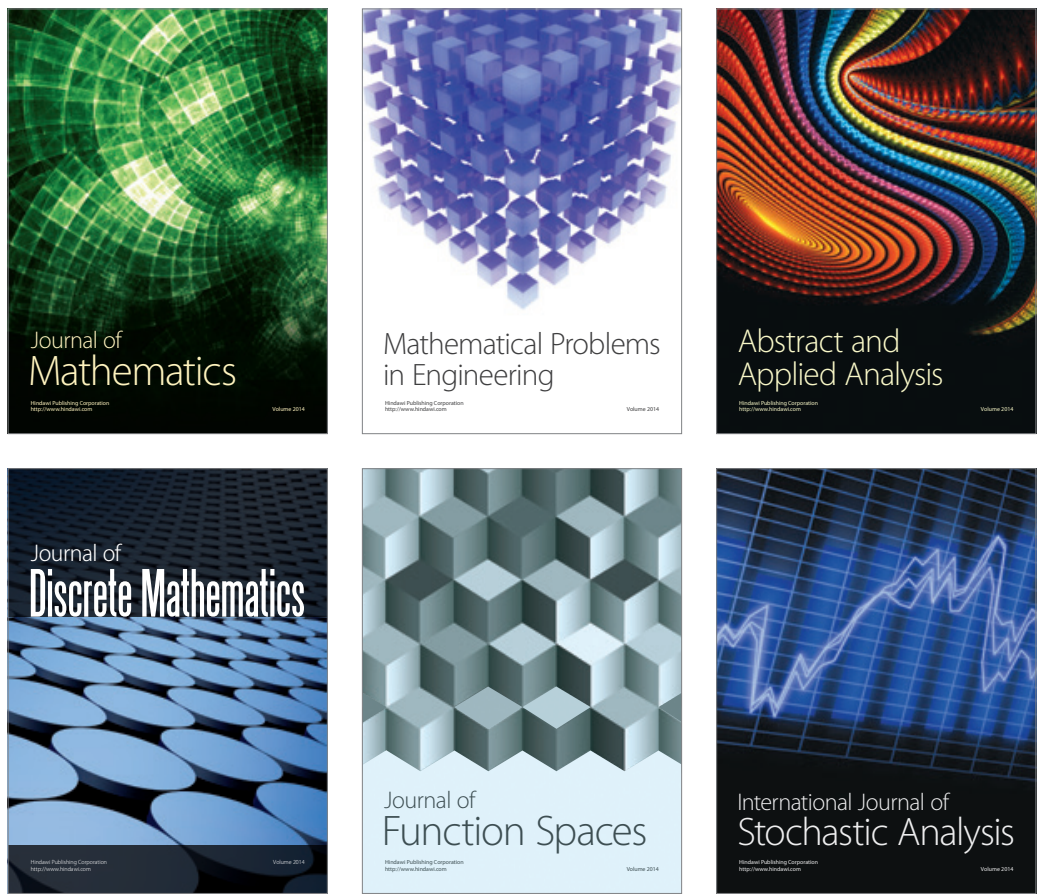

Journal of

Function Spaces

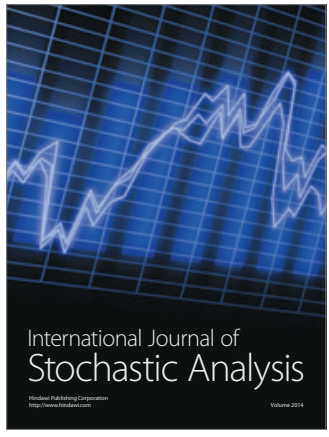

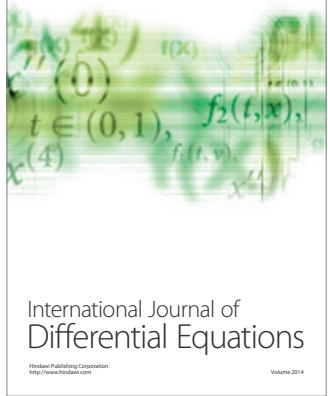
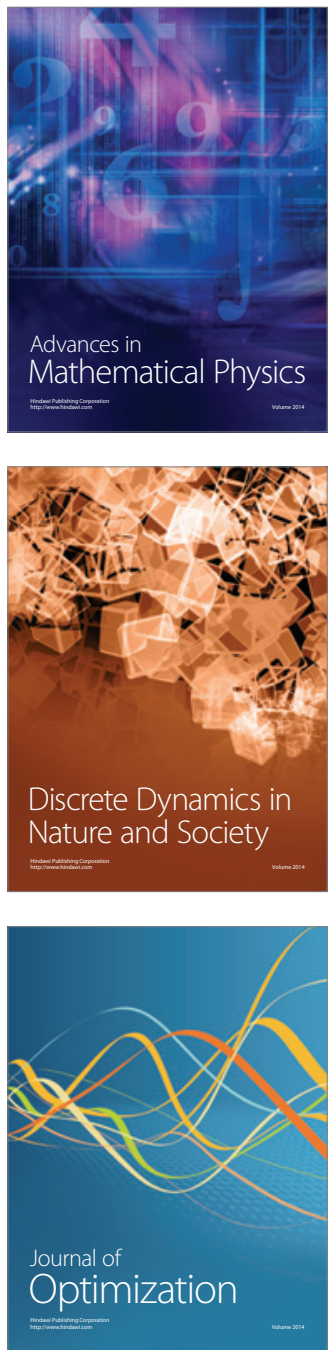